\title{
Beautifying Smile Digitally: A Case Series on Smile Designing with CAD/CAM Veneers
}

\author{
Rajat Lanzara ${ }^{1}$, Mandamparambil Viswambaran $^{2}$, Amit Khattak $^{3}$, Anup Gopi ${ }^{4}$
}

\begin{abstract}
Smile is a person's greatest beauty asset, but various dental diseases like spacing, diastema, discoloration and staining of teeth can adversely affect smile. Literature suggests several approaches for correction of such defects, such as orthodontic intervention, direct and indirect restorative treatments, or their combination. Indirect restorations have been used as a successful option for beautifying smile. But the success of these procedures is based on the correct diagnosis, treatment planning, technique and restorative material used. This article describes three cases with generalized spacing between teeth, diastema and generalized fluorosis which were rehabilitated using lithium disilicate indirect restorations. Digital smile design software was used for diagnosis and treatment planning. These results were used as a guide for designing and fabrication of laminate veneers using CAD/CAM.

Keywords: CAD/CAM, Digital, Esthetics, Laminates, Smile design.

International Journal of Prosthodontics and Restorative Dentistry (2019): 10.5005/jp-journals-10019-1241
\end{abstract}

\section{BACKGROUND}

Teeth presenting with discoloration, diastema and malalignment are a common finding in the anterior maxillary dentition, and this fact can strongly affect the appearance of a smile, besides being displeasing for the patients. ${ }^{1}$ The current literature suggests several approaches for the correction of diastemas and misaligned teeth, such as orthodontic intervention, direct and indirect restorative treatments, or their combination. ${ }^{1-3}$ The restorative treatment is dependent on the size of the diastemas and teeth misalignment, ${ }^{4,5}$ and the success of these procedures is based on the correct diagnosis, planning, technique and restorative material used. ${ }^{6}$ Among the several techniques available for these cases, the most commonly employed are those using direct composite resin or indirect ceramic restorations. Direct restoration with composite resin has advantages such as conservation of tooth tissue, low cost, reversibility, besides being a relatively simple technique. ${ }^{5}$ However, indirect techniques could have a conservative preparation to receive laminated ceramic veneers that present excellent optical properties, compressive strength, surface smoothness, abrasion resistance, gloss and color stability. ${ }^{7}$ The use of indirect restorations is further facilitated with the CAD/CAM technologies, which reduce chair-side time and laboratory steps. ${ }^{8}$ The evolution of CAD/CAM systems has stimulated the development of new ceramic materials, guiding the choice based on the translucency, brightness value, available space in the buccal corridor and the degree of tooth discoloration to optimize the esthetic results. ${ }^{6-9}$ Another important factor to be considered is to plan and estimate the final result that can be achieved in relation to the shape of the teeth, the adaptation of the prosthesis, and the size and the color of the new elements in relation to the soft tissue, lips, and the whole face are very important in the decision-making. ${ }^{10}$ Digital smile design software are a remarkable tool in smile designing that can strengthen diagnostic vision, improve communication, and enhance predictability throughout treatment.

This article describes the complete workflow of beautifying smiles in a digital way by using digital smile design software for evaluation, diagnosis, treatment planning and using CAD/CAM for fabrication of lithium disilicate veneers to achieve the desired esthetic results.
${ }^{1-4}$ Department of Prosthodontics and Crown and Bridge, Army Dental Centre (R \& R), New Delhi, India

Corresponding Author: Rajat Lanzara, Department of Prosthodontics and Crown and Bridge, Army Dental Centre (R \& R), New Delhi, India, Phone: +91 9891817094, e-mail: drrajatlanzara@gmail.com

How to cite this article: Lanzara R, Viswambaran M, Khattak A, et al. Beautifying Smile Digitally: A Case Series on Smile Designing with CAD/CAM Veneers. Int J Prosthodont Restor Dent 2019;9(3):93-98.

Source of support: Nil

Conflict of interest: None

\section{Case Description}

\section{Case 1}

A 23-year-old female patient presented with a complaint of spacing and staining in her upper front teeth and desired to have her teeth esthetically corrected. There was no relevant history contributory to the existing diastema and all other routine investigations were within normal limits. On intra oral examination, the patient was having midline diastema of $4 \mathrm{~mm}$ with mild generalized fluorosis. Overjet and overbite were normal. The patient presented with sound periodontal conditions and no decay was present. On comparing size of central incisors with lateral incisors it was found that proportion was mismatched because of smaller size of central incisors. Treatment options given to patient were orthodontic closure of space and closure of space by porcelain laminate veneers (PLV). Patient opted for PLV considering the shorter duration of treatment protocols and less number of appointments. The treatment protocol is explained in the subsequent paragraphs after brief description of other cases.

\section{Case 2}

A 21-year-old female reported with a chief complaint of impaired smile because of spacing between her upper teeth. Clinical examination revealed class I malocclusion, Elli's class I fracture in crown 21 and generalized fluorosis with spacing between teeth in

(-) The Author(s). 2019 Open Access This article is distributed under the terms of the Creative Commons Attribution 4.0International License (https://creativecommons. org/licenses/by-nc/4.0/), which permits unrestricted use, distribution, and non-commercial reproduction in any medium, provided you give appropriate credit to the original author(s) and the source, provide a link to the Creative Commons license, and indicate if changes were made. The Creative Commons Public Domain Dedication waiver (http://creativecommons.org/publicdomain/zero/1.0/) applies to the data made available in this article, unless otherwise stated. 
the maxillary anterior region. The overjet and overbite were 3 and $2 \mathrm{~mm}$, respectively. There was full complement of teeth with normal attachment of the midline frenum. Oral hygiene was satisfactory. Radiographic examination ruled out presence of any unerupted supernumerary tooth or mesiodens. Various treatment alternatives such as fixed or removable orthodontic appliances were discussed in detail. However, due to prolonged treatment and esthetic issues related to these appliances, patient did not give her consent to these treatment modalities. The space was managed with PLVs after getting an informed consent.

\section{Case 3}

A 28-year-old male patient presented with chief complaint of spacing in upper front teeth and desired to get the space closed. There was no relevant history contributory to the existing diastema and all other routine investigations were within normal limits. On intra oral examination, patient was having midline diastema of $4 \mathrm{~mm}$. Overjet and overbite was reduced due to attrition of maxillary central incisors. The patient presented with sound periodontal conditions and no decay present. Treatment options given to patient were orthodontic closure of space and closure of space by PLVs. Patient opted for PLVs considering the shorter duration of treatment protocols and less number of appointments. So CAD/ CAM made veneers on 11 and 21 were planned to close the existing diastema.

\section{TREATMENT PROTOCOL}

The clinical and laboratory protocols carried out were the same for all the cases. During the initial appointment, diagnostic impressions were made using irreversible hydrocolloid (Algitex, DPI India) and study models retrieved for a comprehensive treatment planning. At this stage a frontal view photograph was transferred to GPS Smile design software (3D smile designer) (Fig. 1A). Considering the patient's facial profile, position of gingiva, contour of lips, and buccal corridor size multiple modules were offered by the software. The modules were tried and superimposed on patient's face to visualize and plan the final outcome of the treatment. The module which best matched the patient's face was selected after due concurrence with patient and his/her parents (Fig. 1B). The digital smile design software gives an idea of amount of tooth reduction and build up required in each tooth to get the desired cosmetic result (Fig. 1C). Mock up preparation of the same was carried out on the model using wax (Fig. 1D) and also intra orally using light cured composite restoration (Fig. 1E). Smile design was carried out, explained to the patient and after getting informed consent, the treatment protocols were started.

At the onset of the treatment, thorough scaling and polishing was done. Before proceeding for tooth preparation, shade was selected using Vitapan classical shade guide (Vita Zahnfabrik, Germany). The veneer preparations started with placement of depth cuts (Fig. 2A). The veneer margins were then established using long, tapered medium grit diamond to prepare definitive chamfer 0.3-0.4 $\mathrm{mm}$ deep at the gingival margin. Tooth contacts were removed and the entire gingivo-proximal definitive chamfer margins were established. Facial preparation was then done for around $1 \mathrm{~mm}$ for facial enamel. The incisal edges were reduced $1 \mathrm{~mm}, 30$ toward the lingual surfaces in relation to 11 and 21 . A final impression was made using a two-step polyvinyl impression technique (Affinis, Coltene Whaledent) (Fig. 2B) and models were poured (Fig. 2C) and die preparation was done (Fig. 2D). Provisional restorations were fabricated using direct light cure composite with spot etching. Scanning of the maxillary and mandibular casts was done (Fig. 3A) and designing of the veneers was done as per the guideline from GPS digital smile design software (Fig. 3B). After removing all undercuts, the restorations were viewed with $3 \mathrm{D}$ reconstruction. Final milling was carried out in glass-ceramic lithium disilicate

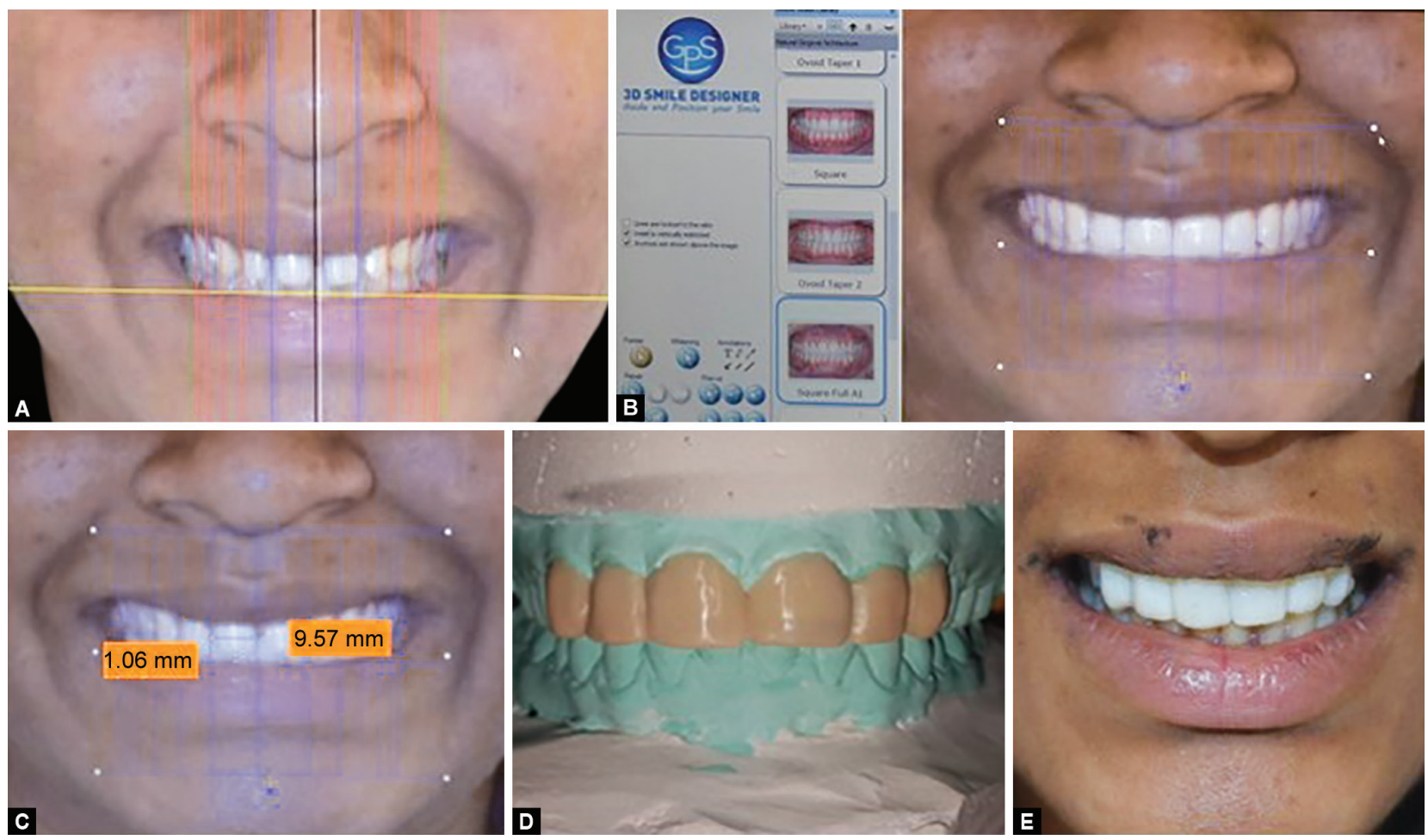

Figs 1 A to E: (A) Frontal view photograph transferred to software; (B) Selection of the mold; (C) Measurements of tooth reduction and buildup required; (D) Mock up on cast; (E) Mock up in situ 

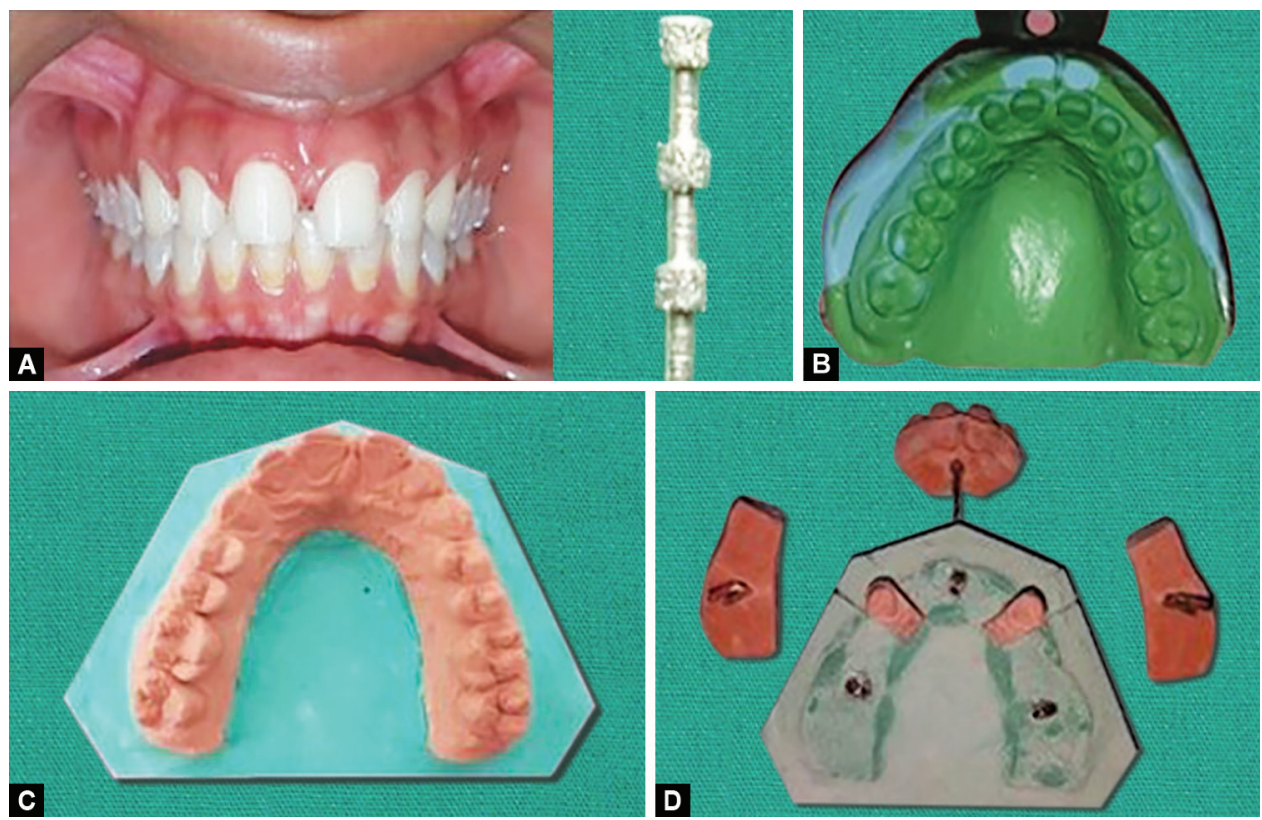

Figs 2A to D: (A) Placement of depth orientation grooves and preparation for porcelain laminate veneer; (B) Polyvinyl siloxane impression; (C) Master cast; (D) Die preparation
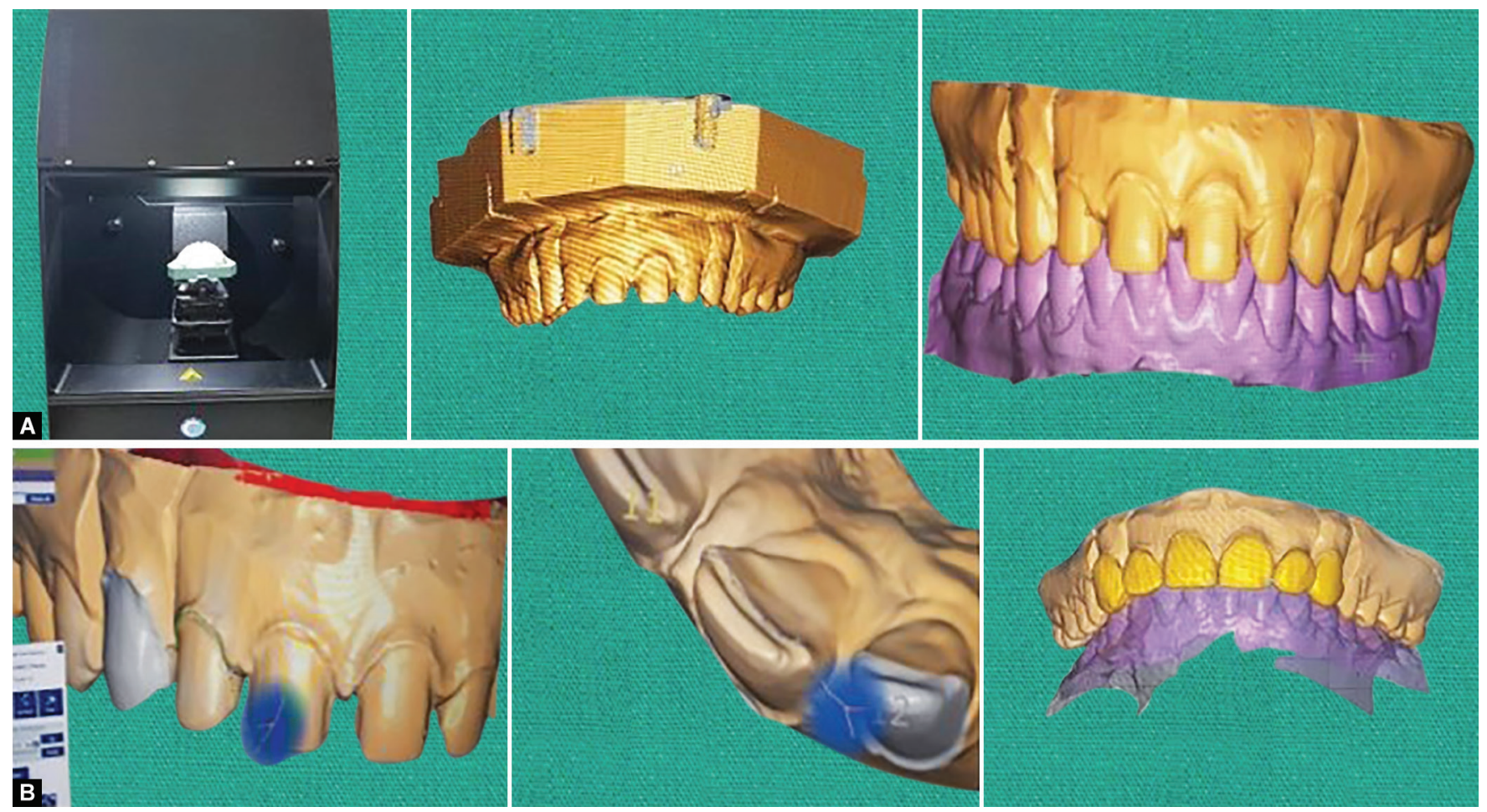

Figs $3 A$ and B: (A) Scanning of maxillary and mandibular casts; (B) Designing of prosthesis in CAD/CAM

(Vita Blocs Triluxe Forte, AmannGirrbach) for fabrication of PLV in the lab (Fig. 4).

The milled veneers were tried on the cast to check for fit and accuracy (Fig. 4C). The internal surfaces of the veneers were etched with $9.5 \%$ hydrofluoric acid (Ultradent, Germany) for 20 seconds (Fig. 4D) and the veneers were silanized with a silane coupling agent (Monobond Plus, Ivoclar Vivadent) before luting. The tooth surface was cleaned using slurry of pumice and gingival retraction cord was placed to control the gingival crevicular fluid. Acid etching of the teeth was done with $37 \%$ phosphoric acid applied for 15 seconds (Total Etch, Ivoclar Vivadent) and the etchant was thoroughly rinsed off. All the teeth surfaces and inner surface of veneers were coated with bonding agent in thin layer and light polymerized for 25-30 seconds. Dual cure composite luting agent (Variolink-II, Ivoclar) of appropriate shade was selected and placed in the laminates. These were placed on the teeth surfaces, 


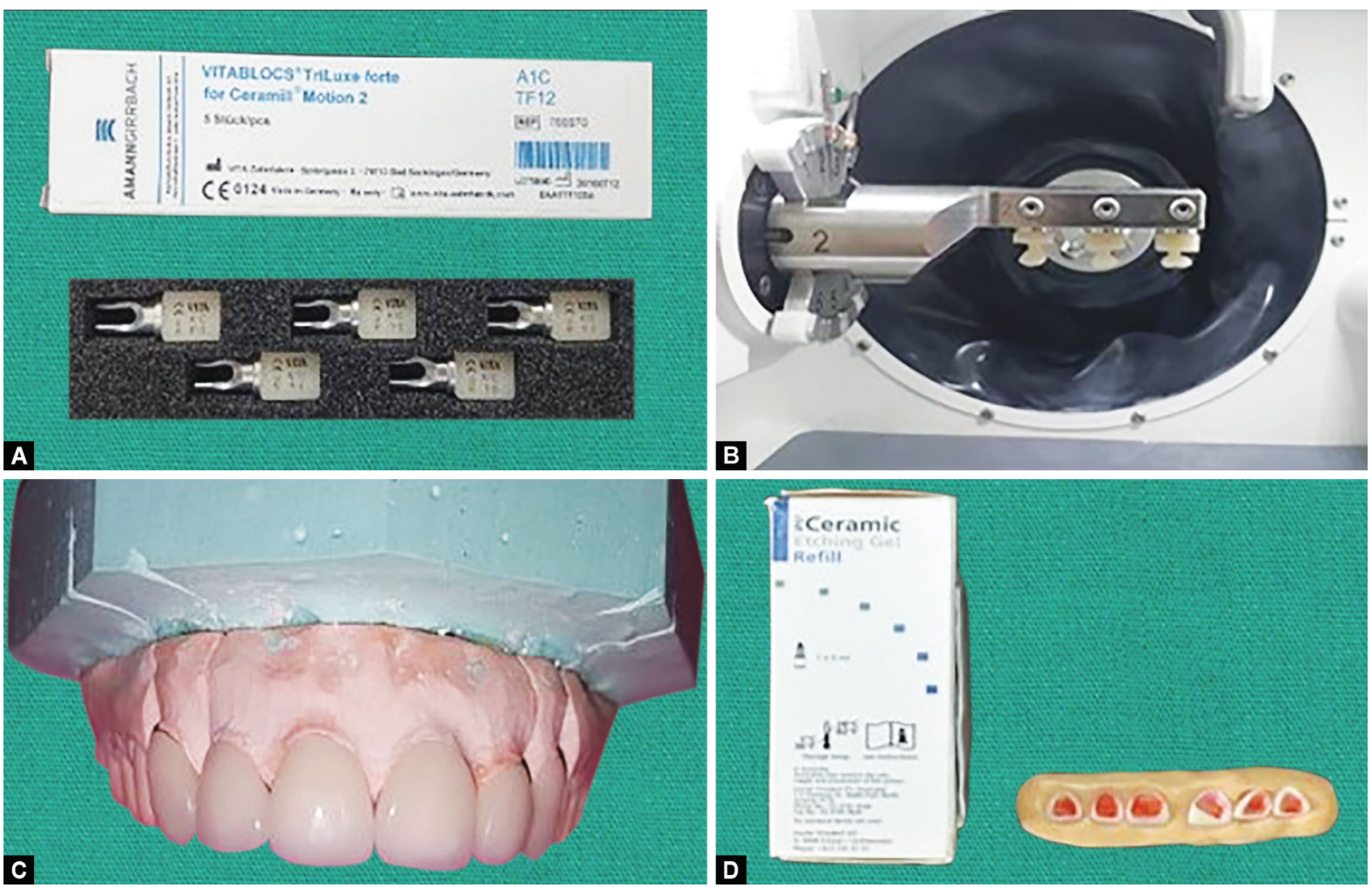

Figs 4A to D: (A) Selection of material for fabrication of prosthesis; (B) Milling procedure; (C) Veneers on the master cast; (D) Etching of ceramic veneers
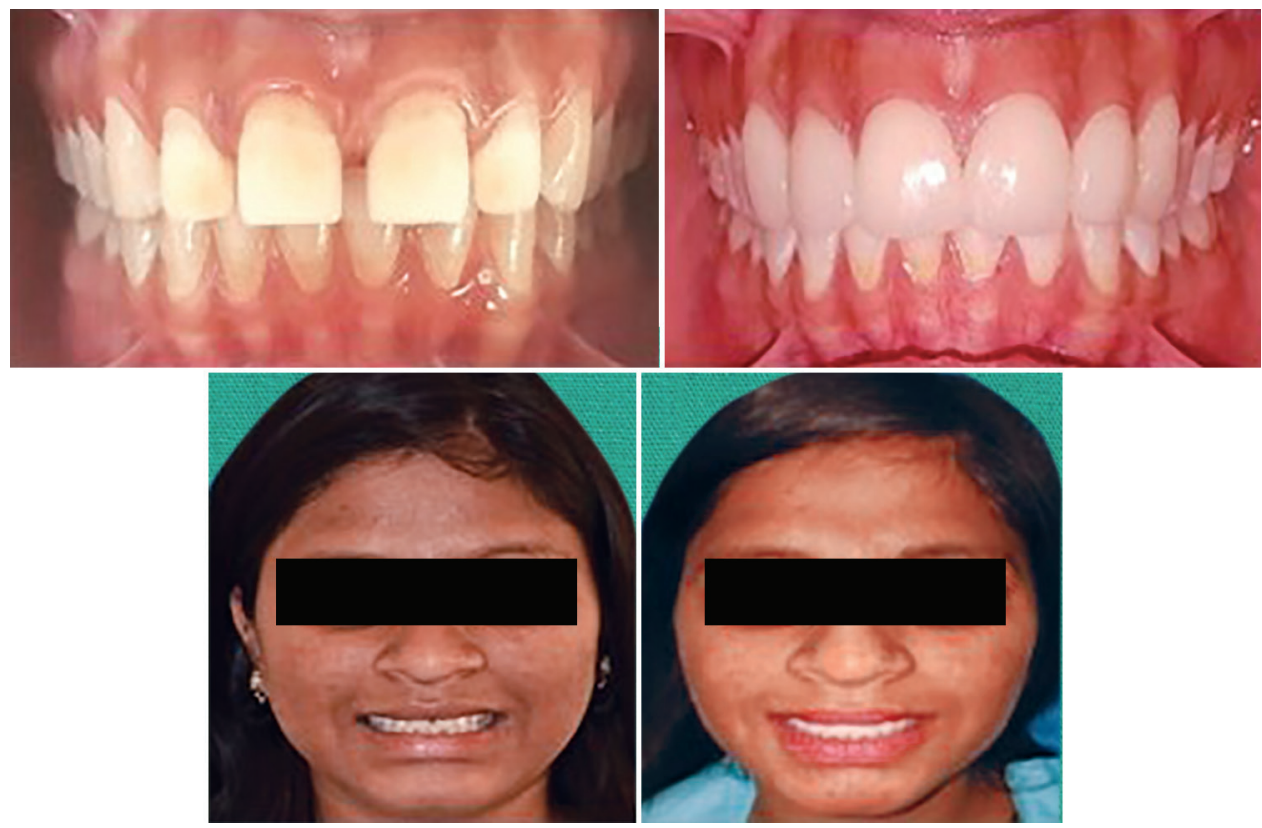

Fig. 5: Comparison of pre- and post-rehabilitation views of Case 1

margins were checked for proper seating, pressure was applied and initial polymerization was done for 5 seconds to remove excess luting agent and cured for 60 seconds and this was done on each tooth. Extra-fine diamond points were used to refine the margins. This treatment procedure had a significant improvement to the patients smile and overall self-esteem as evidenced by the comparison of pre and post rehabilitation photographs. Case 1 is shown in Figure 5, case 2 in Figure 6 and case 3 in Figure 7, respectively.

\section{Discussion}

CAD/CAM technology has been a recently incorporated technology in dentistry, reducing the time of fabrication of ceramic restorations. ${ }^{11,12}$ In this case series the restoration of the tooth is initiated by digitalization of the smile design process. This information is then tested using the mock-up phase of treatment planning. The final ceramic restorations are fabricated such that they replicate the design from chosen module. Lithium 

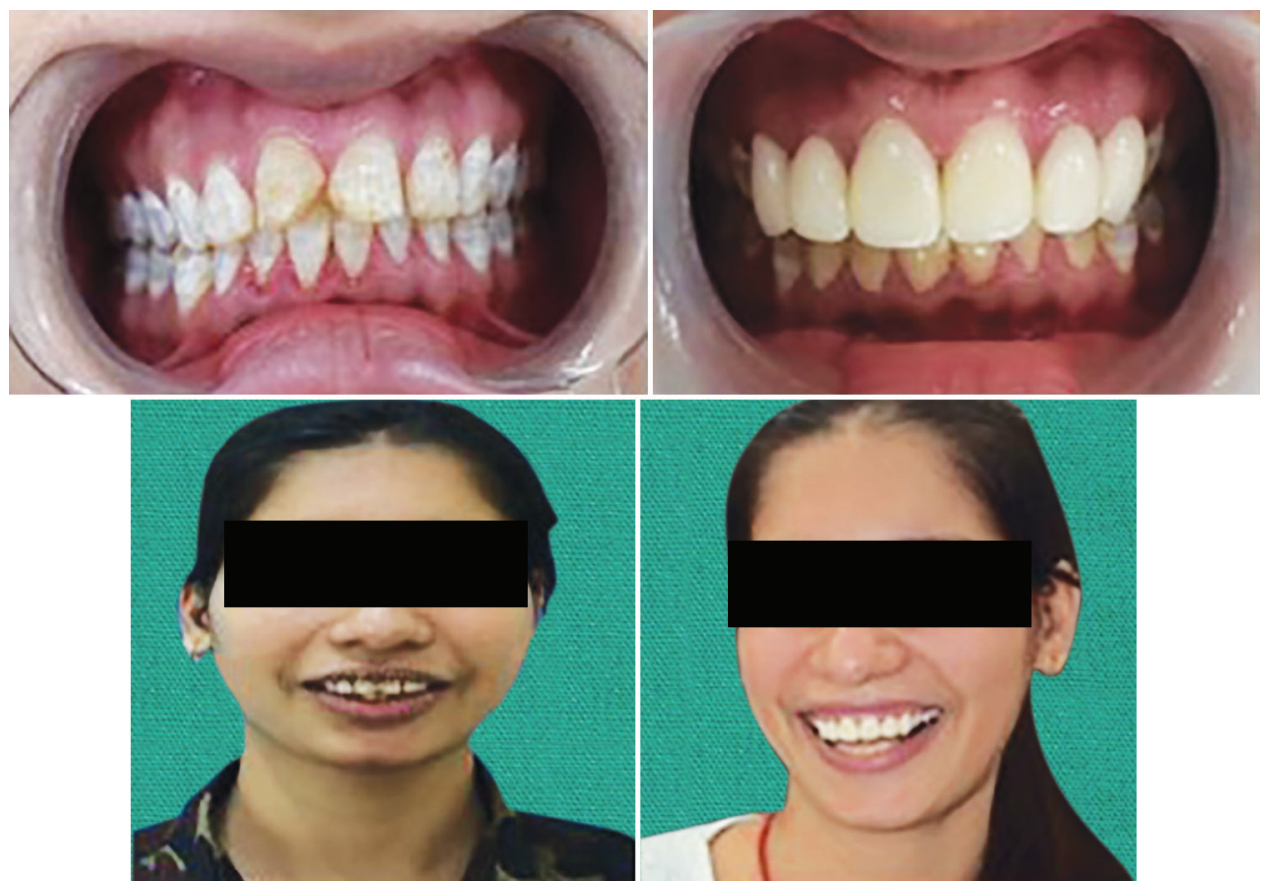

Fig. 6: Comparison of pre- and post-rehabilitation views of Case 2
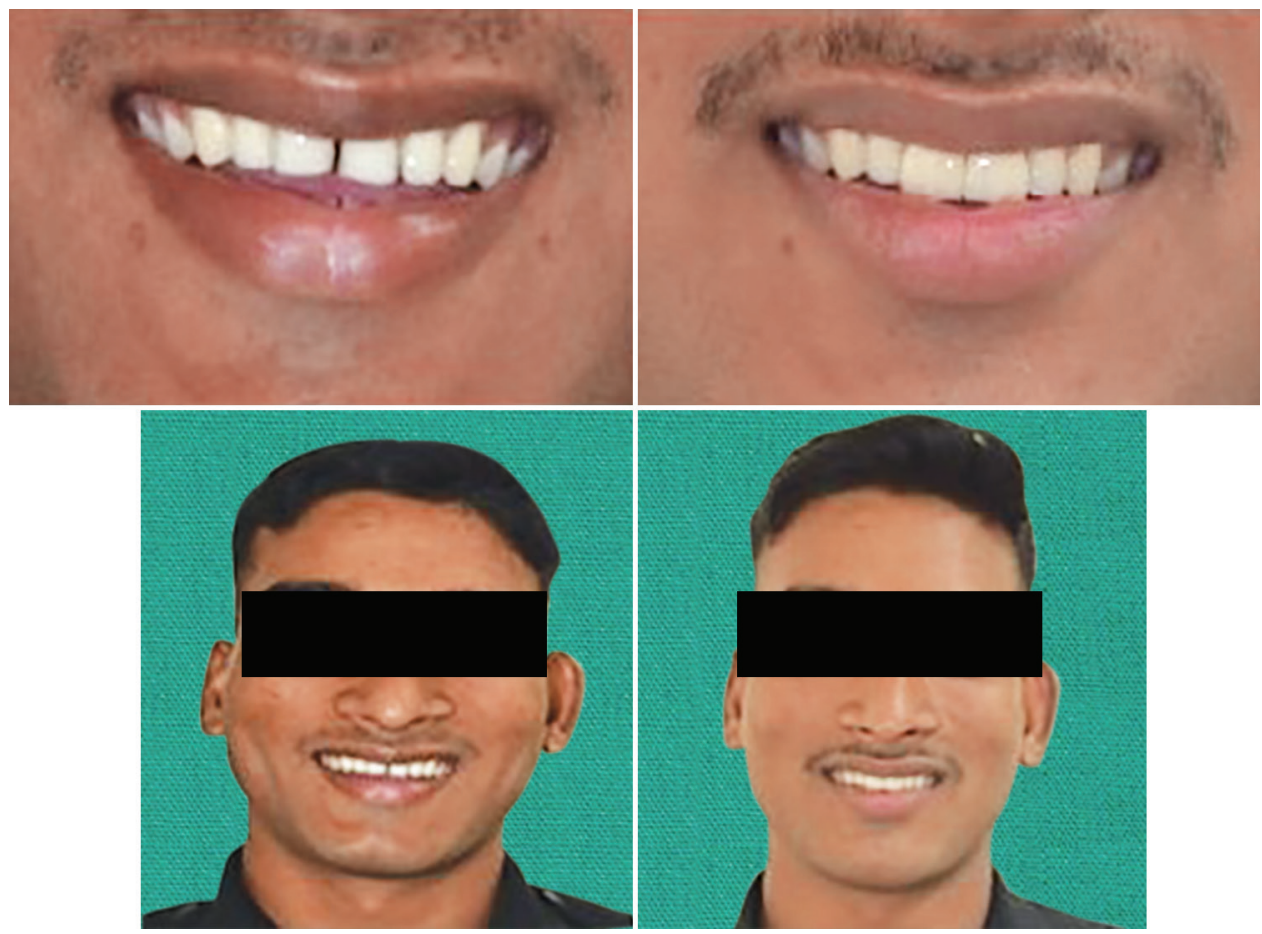

Fig. 7: Comparison of pre- and post-rehabilitation views of Case 3

disilicate ceramic veneers processed through CAD/CAM systems are monolithic restorations, ${ }^{13}$ which means that the final shape of the restoration will be obtained in a single ceramic material which usually lacks the characteristic effects of the anterior dentition such as opalescence, counter-opalescence, white spots, mamelons, etc. But in this case series the prostheses were fabricated using Vita Blocs Triluxe Forte (AmannGirrbach system) which reduced the number of lab steps in fabrication of ceramic veneers. Also the material is available in variety of translucency blocks (HT, high translucency; LT, low translucency; and MO, medium opacity), colors (20 colors) and two shapes of blocks. So it offers several restorative possibilities for rehabilitation of the anterior teeth. According to the manufacturer the flexural strength of the final laminate veneer restoration is approximately $360 \mathrm{MPa},{ }^{14}$ which is sufficient for successful functional rehabilitation as seen in our case series. $^{15}$ 
Porcelain laminate veneers are successful alternatives to direct veneered composite restorations or traditional porcelain-fused-to-metal. Smile can be transformed conservatively, quickly with painless, long-lasting results with the successful use of the PLV. Tissue response is excellent, and the finished surface is very similar to the natural tooth. Veneers exhibit natural fluorescence and absorb, reflect, and transmit light exactly as does the natural tooth structure. The subsequent introduction of special acid etching techniques has improved the long-term retention of veneers. Simonsen and Calamia demonstrated that the bond strength of hydrofluoric acid-etched and silanated veneer to the luting resin composite is generally greater than the bond strength of the same luting resin to the etched enamel surface. ${ }^{16}$ The estimated survival probability of PLVs over a period of 10 years is $91 \%{ }^{17}$

\section{ConcLusion}

The technique described was used to treat various pathologies such as fluorosis, intrinsic stains and spacings in the anterior teeth affecting the cosmetic appearance. The presented case series justifies the choice of beautifying smile using digital smile designing and CAD/CAM veneers, because all the patients had difficulty in social interaction due to poor appearance and requested for immediate results. Digital technology assisted in proper diagnosis, case selection, treatment planning and accurate fabrication which was essential for desired esthetic results.

\section{References}

1. Signore A, Kaitsas V, Tonoli A, et al. Sectional porcelain veneers for a maxillary midline diastema closure: a case report. Quintessence Int 2013;44(3):201-206.

2. Oquendo A, Brea L, David S. Diastema: correction of excessive spaces in the esthetic zone. Dent Clin North Am 2011;55(2):265-281. DOI: 10.1016/j.cden.2011.02.002.
3. De Andrade OS, Ferreira LA, Hirata R, et al. Esthetic and functional rehabilitation of crowded mandibular anterior teeth using ceramic veneers: a case report. Quintessence Int 2012;43(8):661-670.

4. Baratieri LN, Araujo EM, Monteiro S. Composite restorations in anterior teeth: fundamentals and possibilities Quintessence; 2005. pp. 361-367.

5. Soares PV, Spini PH, Carvalho VF, et al. Esthetic rehabilitation with laminated ceramic veneers reinforced by lithium disilicate. Quintessence Int 2014;45(2):129-133.

6. Fehmer V, Mühlemann S, Hämmerle $\mathrm{CH}$, et al. Criteria for the selection of restoration materials. Quintessence Int 2014;45(9):723-730.

7. De Andrade OS, Hirata R, Celestrino M, et al. Ultimate Ceramic Veneer: A Laboratory-Guided Preparation Technique for Minimally Invasive Laminate Veneers. J Calif Dent Assoc 2012;40(6):489-494.

8. Miyazaki T, Hotta Y, Kunii J, et al. A review of dental CAD/CAM: current status and future perspectives from 20 years of experience. Dent Mater J 2009;28(1):44-56. DOI: 10.4012/dmj.28.44.

9. Edelho D, Sorensen JA. Light transmission through all-ceramic dependent on luting material. J Dent Res 2012;81:231-234.

10. Simon $\mathrm{H}$, Magne P. Clinically based diagnostic wax-up for optimal esthetics: the diagnostic mock-up. J Calif Dent Assoc 2008;36(5):355-362.

11. Rani S, Devi J, Jain C, et al. Esthetic rehabilitation of anterior teeth with copy-milled restorations:a report of two cases. Case Rep Dent 2017; 1-5. DOI: 10.1155/2017/2841398.

12. Singh $M$, Mohan $M, C h o u d h a r y S$, etal. "Digitization in prosthodontics,". Int J Dent Health Sci 2014;1(2):208-219.

13. Zarone F, Ferrari M, Mangano FG, et al. "Digitally oriented materials": Focus on lithium disilicate ceramics. Int J Dent 2016;36(5):355-362. DOI: 10.1155/2016/9840594.

14. Beuer F, Schweiger J, Edelhoff D. Digital dentistry: an overview of recent developments for CAD/CAM generated restorations. Br Dent J 2008;204(9):505-511. DOI: 10.1038/sj.bdj.2008.350.

15. Jevremovic D, Trifkovic B, Lapcevic A, et al. The use of CAD/CAM technology in design and manufacture of thin laminate veneers. $J$ Manuf Ind Eng 2012;11(3):54-56.

16. Calamia JR, Simonsen RJ. Effect of coupling agents on bond strength of etched porcelain. J Dent Res 1984;63:179-183.

17. Frese C, Staehle HJ, Wolff D. The assessment of dentofacial esthetics in restorative dentistry: a review of the literature. J Am Dent Assoc 2012;143:461-466. DOI: 10.14219/jada.archive.2012.0205. 